IMSc $/ 98 / 10 / 51$

hep-th/9810069

\title{
The Hagedorn Transition, Deconfinement and the AdS/CFT Correspondence
}

\author{
S. Kalyana Rama and B. Sathiapalan \\ Institute of Mathematical Sciences \\ Taramani, Chennai 600113, INDIA
}

June 28, 2021

\begin{abstract}
A connection between the Hagedorn transition in string theory and the deconfinement transition in (non-supersymmetric) Yang-Mills theory is made using the AdS/CFT correspondence. We modify the model of zero temperature QCD proposed by Witten by compactifying an additional space-time coordinate with supersymmetry breaking boundary conditions thus introducing a finite temperature in the boundary theory. There is a Hagedorn-like transition associated with winding modes around this coordinate, which signals a topology changing phase transition to a new AdS/Schwarzschild blackhole where this coordinate is the time coordinate. In the boundary gauge theory time like Wilson loops acquire an expectation value above this temperature.
\end{abstract}




\section{Introduction}

The question of whether the Hagedorn temperature [1] is really a limiting temperature or whether it is to be interpreted as a phase transition temperature is not fully understood. One motivation for interpreting it as a phase transition comes from the belief that large-N Yang-Mills theory has a string description, where the string coupling constant, $g_{s}$, can be identified with $1 / N$. This theory is believed to have a transition (for any $N$ ) from a low temperature confining phase where the lightest degrees of freedom are colour singlets, to a high temperature deconfined phase where the light degrees of freedom are gluons (and quarks). It is only natural to identify the Hagedorn temperature with the temperature of the deconfinement transition. However the nature of the phase above that temperature is not fully understood. In a recent work by one of us [2], using a matrix model description a picture of the phase transition was obtained that lends credence to the identification of the Hagedorn transition as signalling something like a deconfinement transition. It was found that at high temperatures D-0-branes tend to cluster and the uniform distribution that is necessary in order to form a string is destroyed. The transition from uniform distribution to clustering is very similar to that found in gauge theories at the deconfinement transition temperature. The expectation value of the time-like Wilson loop $<\operatorname{Tr} e^{i \int A_{0} d t}>$ vanishes when the eigenvalues of $\int A_{0} d t$ are uniformly distributed and becomes non-zero when the eigenvalues cluster. Thus the high temperature phase is a cluster of D-0-branes.

One can ask this question again in the light of some of the recent developments that relate string theory in AdS backgrounds with supersymmetric gauge theories [[3]-[9]]. Here, even more than in matrix theory, the connection between gauge theories and string theory is very direct. Particularly useful is the fact that the expectation value of a Wilson loop is given by summing over string world sheets that have this loop as their boundary [6, 7].

In [5] a proposal was made to study non-supersymmetric QCD in this approach. Several qualitative features expected in QCD were demonstrated such as the area law for Wilson loops and the existence of a mass gap. Following this proposal masses of the glueball states were computed and reasonable agreement was found with lattice calculations [10, 11, 12]. It has also been pointed out that the AdS/CFT correspondence has been tested

in the regime where $g^{2} N$ is large and therefore cannot be said to describe 
continuum QCD but rather QCD on a very coarse lattice. In this regime one cannot expect any kind of universality, i.e. the details of the bare theory and the regularization become important. In particular one should not expect all physical quantities to be some pure number (dependent only on $N$ ) times some appropriate power of $\Lambda_{Q C D}$. Presumably this explains the factor of $g^{2} N$ relating the string tension to glueball mass [13]. Furthermore it is not clear that the continuum theory that one can get from this is the usual QCD or QCD along with an infinite number of other particles coming from the Kaluza Klein states [9, 14]. Ignoring these problems for the moment one can go ahead and address the issue of the connection between the Hagedorn transition and deconfinememt. I Various issues concerning the Hagedorn transition have been discussed in recent papers [16, 17].

Our starting point is the proposal of [5] for studying non-supersymmetric QCD. Non-extremal M-5 branes in Euclidean $A d S_{7} \times S_{4}$ are described by the following metric: $\left(u, x^{i}\right.$ are dimensionless. $l_{11}$ is the Planck length in eleven dimensions.)

$$
\begin{gathered}
\frac{d s^{2}}{\left(l_{11}\right)^{2}}=\frac{u^{2}}{(\pi N)^{1 / 3}} \sum_{i=1}^{5}\left(d x^{i}\right)^{2}+ \\
\frac{u^{2}}{(\pi N)^{1 / 3}}\left(1-\frac{u_{0}^{6}}{u^{6}}\right) d x_{0}^{2}+4(\pi N)^{2 / 3} \frac{1}{\left(1-\frac{u_{0}^{6}}{u^{6}}\right)} \frac{d u^{2}}{u^{2}} \\
+(\pi N)^{2 / 3} d \Omega_{4}^{2}
\end{gathered}
$$

The time direction is compactified with a period $\frac{4 \pi}{3} \sqrt{\frac{\pi N}{u_{0}^{2}}}$ and supersymmetry is broken by the finite temperature boundary conditions. One more direction is compactified with supersymmetric boundary conditions. Thus one ends up with a limiting form of an AdS Schwarzschild black hole or non extremal D4 brane metric with one compact Euclidean time direction to give a four (non compact Euclidean) dimensional boundary gauge theory with massive fermions (supersymmetry is broken). This theory is 4-dimensional Euclidean QCD. By the rescaling $\frac{u}{u_{0}}=\frac{\rho}{b}$ with $b=(\pi N)^{1 / 3} l_{11}$ and $\frac{u_{0}}{\sqrt{\pi N}} x^{i}=z^{i} \mathrm{i}=1, . .4$; $x^{5}=\frac{R_{11}}{l_{11}} \theta$ and $\phi=\frac{3 u_{0}}{2 \sqrt{\pi N}} x_{0}$, one ends up with the form $\left(R_{11}, b\right.$ have dimensions of length and so $\rho$ has dimensions of length and all other coordinates

\footnotetext{
${ }^{1} \mathrm{~A}$ deconfinement transition is expected in the lattice strong coupling theory also 18 . So this is still a pertinent question.
} 
are dimensionless.)

$$
d s^{2}=\frac{4}{9} \rho^{2}\left(1-\frac{b^{6}}{\rho^{6}}\right) d \phi^{2}+\frac{4 b^{2}}{\left(1-\frac{b^{6}}{\rho^{6}}\right)} \frac{d \rho^{2}}{\rho^{2}}+\rho^{2} \sum_{i=1}^{4}\left(d z^{i}\right)^{2}+b^{2} d \Omega_{4}^{2}+\frac{u_{0}^{2} R_{11}^{2}}{\pi N l_{11}{ }^{2}} \rho^{2} d \theta^{2}
$$

where $\theta$, the "eleventh" coordinate and $\phi$ the Euclidean time/temperature coordinate have periods $2 \pi$. If we ignore $\theta$, (2) represents a 10-dimensional space-time appropriate for string theory. The dilaton field satisfies $e^{2 \phi / 3}=$ $\frac{R_{11} u}{l_{11(\pi N)^{1 / 6}}}$. The 'string metric' where $l_{s}$ is treated as a constant can be written as follows: $\left(w=g_{s}^{1 / 3} u^{2}\right.$ and $\left.y^{i}=x^{i} g_{s}^{1 / 3}\right)$ ?

$\frac{d s^{2}}{l_{s}{ }^{2}}=\frac{w^{3 / 2}}{\sqrt{g_{s} N \pi}}\left(1-\frac{w_{0}^{3}}{w^{3}}\right) d y_{0}^{2}+\frac{w^{3 / 2}}{\sqrt{g_{s} N \pi}} \sum_{i=1}^{4}\left(d y^{i}\right)^{2}+\frac{\sqrt{g_{s} N \pi}}{w^{3 / 2}\left(1-\frac{w_{0}^{3}}{w^{3}}\right)} d w^{2}+\sqrt{g_{s} N \pi w} d \Omega_{4}^{2}$

Note that while there is a temperature $\left(=\frac{3}{4 \pi} \sqrt{\frac{w_{0}}{g_{s} N}}\right)$ associated with the black hole and we are doing finite temperature string theory, from the viewpoint of the gauge theory we are still at zero temperature. The time ccordinate of the black hole metric is not one of the four coordinates $y^{i}, i=1, . .4$ of the gauge theory. We, however, would like to study the gauge theory at finite temperature. Accordingly we compactify one of the Euclidean directions of the gauge theory (say $z_{4}={ }^{\prime} z^{\prime}$ ) and thus also of string theory, with supersymmetry breaking boundary conditions. The radius $R_{z}$ of this circle is $\frac{\beta}{2 \pi}$, where $\beta$ is the inverse temperature of the gauge theory. \& ¿From the viewpoint of string theory we have a compactification of Type II string theory with one compact direction ' $z$ ' for supersymmetry breaking (first studied in [15]) at finite temperature. Thus the proposal of [5] is modified in that over and above finite temperature effects there is a supersymmetry breaking due to boundary conditions in the $z$-direction. Thus the zero-temperature string theory itself has broken supersymmetry. As shown in 15 this non supersymmetric string has a tachyon in the sector with non zero winding

\footnotetext{
${ }^{2}$ Note that $g_{s}$ is defined as the asymptotic value of $e^{\phi}$ at infinity where space is flat. This is $\left(\frac{R_{11}}{l_{11}}\right)^{3 / 2}$. The expression for the dilaton given above is only valid in the near horizon region where the metric is AdS. Thus asymptotically $\left(l_{11}\right)^{2}=g_{s}^{2 / 3}\left(l_{s}\right)^{2}$, whereas in the AdS region where we are working $\left(l_{11}\right)^{2}=g_{s}^{2 / 3} \frac{u}{(\pi N)^{1 / 6}}\left(l_{s}\right)^{2}$.

${ }^{3}$ We remind the reader that the string theory is already at finite temperature identified with the radius $\frac{2}{3} \sqrt{\frac{g_{s} N}{w_{0}}}$ of the compact Euclidean time coordinate $y_{0}$.
} 
in the $z$-direction when the radius is smaller than a critical value of $O\left(l_{s}\right)$. This is similar to the tachyonic mode found in the time-like direction in finite temperature string theory, signalling the Hagedorn transition [20, 21]. In the (finite temperature) supersymmetric case one had to explain why this tachyon is not projected out by the GSO projection. This was done in [19]. For similar reasons the tachyon in the winding sector in the $z$-direction is not projected out by the GSO projection.

Thus for a critical value of $\beta$ one expects a Hagedorn-like phase transition. We would like to argue that this signals a deconfinement transition in the boundary gauge theory. To make a connection with deconfinement one has to show that the expectation value of the time like Wilson loop (or Polyakov loop) is non-zero, i.e.

$$
\begin{aligned}
& <P(C)>\equiv<\operatorname{Tr} e^{i \int_{c} A_{0} d t}>=0 \quad \beta>\beta_{c} \\
& <P(C)>\equiv<\operatorname{Tr} e^{i \int_{c} A_{0} d t}>\neq 0 \quad \beta \leq \beta_{c}
\end{aligned}
$$

One expects in continuum QCD that $T_{c}$ will be of the same order as the string tension. We will in fact see that the Hagedorn transition is of this order. 1

Let us remind ourselves why $P(C)$ is zero at low temperatures [5]. According to the prescription for calculating Wilson loops one has to sum over surfaces of which this loop $C$ is the boundary. In the following discussion, for convenience we will refer to $y_{0}$ as $t$ and $y_{4}$ as $z$. If we compactify the $z$-coordinate in the usual Kaluza-Klein manner, the $S^{1}$ around that direction is non-simply connected. Thus this $S^{1}$ is not the boundary of any two dimensonal surface that has the topology of a disc. Hence $\langle P(C)\rangle=0$. In order for $\left\langle P(C)>\right.$ to become non-zero at high temperatures, $S^{1}$ must somehow become the boundary of some surface. The space time manifold must change topology for this to happen. Now the time coordinate of a non extremal D-brane black hole is simply connected - if the $S^{1}$ had been around this coordinate it would be the boundary of a two dimensional surface and $P(C)$ would be non-zero. Thus if it so happens that the topology changes

\footnotetext{
${ }^{4}$ The actual $T_{c}$ will turn out to be a little different - in fact it is of the order of the mass of the glueball. Again, as mentioned above the fact that the glueball mass and the string tension are not the same must be an artifact of the fact that we are doing a strong coupling calculation and are far from the continuum limit.
} 
so that $z$ becomes the Euclidean time coordinate (and so $t$ becomes just another space coordinate) $P(C)$ will become non zero. It is plausible that such a tunneling can take place since in Euclidean space all the 10 coordinates are on equal footing and a priori any of them can be analytically continued to a Minkowski time coordinate. In any case one must sum over all bulk manifolds whose boundary is $S^{1} \times S^{1} \times \Sigma_{3}$ spanned by $y^{0}, y^{4}$ and $y^{i}, i=1,2,3$ respectively [4, [5]. Thus it is imperative that these topologies be considered in calculating the partition function. One can compare the free energies of the two configurations i.e. one, say $X_{t}$, where $t$ is time and and the other $X_{z}$ where $z$ is time, and see which configuration is dominant as $R_{z}$ or equivalently the gauge theory temperature $=\frac{1}{2 \pi R_{z}}$ is varied. By symmetry it is clear that there will be an inverse transition temperature $\beta_{c}=2 \pi\left(R_{z}\right)_{c}$ at which $X_{z}$ is preferred and furthermore (again by symmetry) that $\beta_{c}$ must be reached when the two radii $R_{t}$ and $R_{z}$ are equal. We will be more explicit in the next section.

Thus we see that at a critical temperature there will be a phase transition at which $P(C)$ acquires a non-zero vacuum expectation value. ¿From the string viewpoint it is a change of topology of space time. This is the deconfinement transition for the boundary gauge theory. In fact we will see that this critical temperature will be lower than the "Hagedorn" temperature of the $z$-drection. This is just as well. At the Hagedorn temperature, the partition function diverges and therefore the actual phase transition should take place before that. This is also consistent with the results of the weak coupling string perturbation calculation of [19].

\section{Calculation of the Deconfinement Temper- ature}

We will use the metric (2) and assume that the boundary gauge theory is located at some value of $\rho$ say $\rho_{\infty}>>b$. We will also take the boundary metric to be $d s^{2}=\rho_{\infty}^{2} \sum_{i=1}^{4}\left(d z^{i}\right)^{2}$. Thus the value $\rho_{\infty}$ can be thought of as a scale in the renormalization group sense. Using the usual arguments the string tension of the boundary gauge theory is $\sigma=\frac{b^{2}}{\rho_{\infty}^{2} l_{s}^{2}}$. We must remember that $l_{11}=e^{\phi / 3} l_{s}$ and hence in the metric of (2) $l_{s}$ is $u$ dependent. If we go to the string metric we find $\sigma=\left(\frac{w_{0}}{w_{\infty}}\right)^{3 / 2} \frac{1}{l_{s}^{2}}$, where $w_{\infty}$ is the value 
of $w$ of the boundary gauge theory (see (3)). If in (2) we compactify say $z^{4}=z$ with a radius $R_{z}$ with supersymmetry breaking boundary conditions, we get a boundary gauge theory with inverse temperature $\beta=\rho_{\infty} 2 \pi R_{z}$. As we reduce $R_{z}$ the winding mode becomes tachyonic first near $\rho=b$. this happens when $2 \pi R_{z} b \approx l_{s}$. Thus the temperature at the boundary when this Hagedorn-like transition takes place is $\approx \frac{b}{\rho_{\infty} l_{s}} \approx \sqrt{\sigma}$. This is of course when the deconfinement transition is also expected to take place. This supports the idea that a Hagedorn transition in the bulk signifies a deconfinemnt transition in the boundary.

The value of $2 \pi R_{t}$, the circumference of the time coordinate at $w_{\infty}$, is $\frac{4 \pi}{3} \frac{w_{\infty}^{3 / 4}}{\left(g_{s} N\right)^{1 / 4}} \sqrt{\frac{g_{s} N}{w_{0}}} l_{s}=\frac{4 \pi}{3}\left(g_{s} N\right)^{1 / 4} \frac{w_{\infty}^{3 / 4}}{w_{0}^{1 / 2}} l_{s}$. Thus the ratio $\frac{R_{z}}{R_{t}}$ when the Hagedorn transition takes place is $\frac{1}{\left(w_{0} g_{s} N\right)^{1 / 4}}$ which is expected to be less than one as $g_{s} N$ is much greater than one, unless $w_{0}$ is very small. In fact, as $R_{z}$ is reduced (thus raising the gauge theory temperature), before the Hagedorn transition is reached at $R_{z}<R_{t}$, when $R_{z}$ becomes equal to $R_{t}$ we expect a phase transition to a spacetime where $z$ is the time coordinate. This can be easily seen by comparing the corresponding expressions for the free energy [17]. Let $F\left(R_{z}, R_{t}\right)$ be the expression for the free energy when $t$ is the time coordinate (we do not need the exact expression here). When $z$ becomes the time coordinate we get $F\left(R_{t}, R_{z}\right)$ (by symmetry) - the same function with $R_{z}$ and $R_{t}$ interchanged. We know that when $R_{z}$ is infinite, the preferred configuration is the one where $t$ is the time coordinate. Therefore it must be that $F\left(R_{z}, R_{t}\right)<F\left(R_{t}, R_{z}\right)$ when $R_{z}$ is very large. Clearly the transition to the other regime, which we have seen makes $\langle P(C)\rangle$ non zero, occurs when $F\left(R_{z}, R_{t}\right)=F\left(R_{t}, R_{z}\right)$ which means $R_{t}=R_{z}$.

Thus unless $w_{0}$ is very small we expect the deconfinement transition to take place before the Hagedorn. The temperature at which deconfinement happens (i.e. when $R_{z}=R_{t}$ ), is $\frac{1}{2 \pi R_{t}} \approx O\left(M_{\text {glueball }}\right)$ [10, 11, 12]. As mentioned earlier, the fact that this is not equal to the string tension must be an artifact of the strong coupling limit. Presumably if we knew how to go to weak coupling these two scales would converge.

To establish that the entropy does jump by an amount proportional to $N^{2}$, [22, 1], we need to look at the expression for $F$. Actually it is trivially proportional to $N^{2}$ always because of the presence of Newton's constant. But, as explained in [4] the part that is linearly dependent on $\beta$ is just the zero temperature contribution. What we have to look at is the coefficient 
of the piece that has a non trivial $\beta$ dependence. Another way of saying the same thing is that the thermodynamic free energy is $\frac{-\ln Z}{\beta}$ whereas we have been referring to $-\ln Z$ as the free energy. After dividing by $\beta$, the piece that is temperature dependent should be proportional to $N^{2}$ above the deconfinement transition, whereas below the transition this piece will be of $O(1)$. Remembering that $R_{z}$ is $\frac{\beta}{2 \pi}$ we can easily see that $F\left(R_{t}, \frac{\beta}{2 \pi}\right)$ must have the form (in the supergravity approximation, when the fields or metric have no dependence on $\beta) \beta N^{2} f\left(R_{t}\right)$ which is linear in $\beta$ and does not contain any non linear $\beta$ dependence. Thus after dividing by $\beta$ there is no temperature dependent contribution in this approximation. This means in particular that there are no finite temperature effects that are proportional to $N^{2}$. ( We remind the reader that by 'temperature' we mean the temperature of the boundary four dimensional gauge theory and not the temperature of the AdS-Schwarzschild black hole.) The function $F\left(\frac{\beta}{2 \pi}, R_{t}\right)$ on the other hand is of the form $2 \pi R_{t} N^{2} f\left(\frac{\beta}{2 \pi}\right)$ which definitely has a non trivial $\beta$ dependence proportional to $N^{2}$. We do not need the exact expression for $f$ to make these arguments. We only need to realize that it cannot be linear in its argument

and therefore $\frac{f\left(\frac{\beta}{2 \pi}\right)}{\beta}$ is still temperature dependent. This is due to the nontrivial $\beta$-dependence of the metric. The reader can verify this using the exact expression given in 17] for the case at hand. Thus this transition has all the characteristics expected of a deconfinement transition in gauge theories.

\section{Conclusion}

To summarize we have shown that one can relate the deconfinement transition of finite temperature QCD to a Hagedorn-like transition in the bulk string theory. The Hagedorn transition involves condensation of vortices around a compact coordinate ' $z$ ' with supersymmetry breaking boundary conditions. This signals a phase transition where the topology of spacetime changes. The new topology is one where $z$ becomes the time coordinate and the old time coordinate becomes a space coordinate. The expectation value of the Polyakov loop becomes non zero and therefore this transition can be identified with deconfinement.

When we combine the above arguments with the results of [2] the following picture of the deconfinement transition emerges: As one heats up the gauge theory there is a tendency for the D-0-branes making up the mem- 
brane (we are using the matrix model [23] language here) that becomes a string when wound around a compact direction, to cluster. This is because supersymmetry is broken by the boundary conditions on the z-direction. This is explained in [2]. One can think of this as a tendency of the string to shrink to zero size. This makes the $z$-coordinate pinch off to a point so that the string can shrink to zero size at the tip near the region of small $u$ and disintegrate into a bunch of D-0-branes. Thus near (inside?) the horizon we have a cluster of D-0-branes and no string. This disintegration happens at the small $u$ end first because the temperature increases as you move towards the horizon and is highest at the horizon. Just when the $z$-coordinate pinches off and $\langle P(C)>$ becomes non-zero, the $t$-coordinate opens up and becomes an ordinary non-simply-connected circle.

\section{References}

[1] R. Hagedorn, Nuovo Cimento Suppl. 3 (1965) 147.

[2] B. Sathiapalan, Mod. Phys. Lett. A13 (1998) 2085, hep-th/9805126.

[3] J. Maldacena, Adv. Theor. Math. Phys. 2 (1998), hep-th/9711200.

[4] E. Witten, Adv. Theor. Math. Phys. 2 (1998), hep-th/9802150.

[5] E. Witten, hep-th/9803131.

[6] J. Maldacena, Phys. Rev. Lett. 80 (1998) 4859, hep-th/9803002.

[7] S. J. Rey and J. Yee, hep-th/9803001.

[8] S.S. Gubser, I. R. Klebanov and A. M. Polyakov, Phys. Lett. B428 (1998) 105, hep-th/9802109.

[9] D. J. Gross and H. Ooguri, hep-th/9805129.

[10] C. Csaki, H. Ooguri, Y. Oz and J. Terning, hep-th/9806021.

[11] R. de Mello Koch, A. Jevicki, M. Mihailescu and J. P. Nunes, hepth/9806125.

[12] M. Zyskin, hep-th/9806128. 
[13] J. Greensite and P. Olesen, hep-th/9806235.

[14] H. Ooguri, H. Robins and J. Tannenhauser, hep-th/9806171.

[15] R. Rohm, Nucl. Phys. B237 (1984) 553.

[16] S. Chaudhury and D. Minic, Phys. Lett. B433 (1998) 301, hepth/9803120.

[17] J. L. F. Barbon, I.I Kogan, E. Rabinovici, hep-th 9809033.

[18] B. Svetitsky, Phys. Rep. 132 (1986) 1.

[19] J. J. Atick and E. Witten, Nucl. Phys. B310 (1988)291.

[20] B. Sathiapalan, Phys. Rev. D35 (1987)1597.

[21] Ya. I. Kogan, JETP Lett. 44 (1986)267.

[22] C. Thorn, Phys. Lett. 99B(1981)458.

[23] T. Banks, W. Fischler, S. Shenker and L. Susskind, Phys Rev D55(1997) 5112, hep-th/9610043. 